ISSN 1997-342X (Online), ISSN 1991-8631 (Print)

Original Paper http://ajol.info/index.php/ijbcs http://indexmedicus.afro.who.int

\title{
Actualisation de l'entomofaune des cultures maraîchères en Basse Casamance (Sénégal)
}

\author{
Etienne TENDENG ${ }^{1,2, *}$, Babacar LABOU ${ }^{1}$, Saliou DJIBA ${ }^{2}$ et Karamoko DIARRA ${ }^{1}$ \\ ${ }^{1}$ UCAD, Equipe Production et Protection Intégrées en Agroécosystèmes Horticoles - 2PIA, \\ Faculté des Sciences et Techniques, Dakar, Sénégal. \\ ${ }^{2}$ ISRA, Centre de Recherches Agricoles de Djibélor, B.P 34, Ziguinchor, Sénégal. \\ "Auteur correspondant. E-mail: etienne.tendeng@ucad.edu.sn letiennetendeng@gmail.com; \\ Tel : +221-77-701-04-09
}

\section{REMERCIEMENTS}

Nous exprimons nos sincères gratitudes à l'Institut Sénégalais de Recherches Agricoles et au Programme de Productivité Agricole en Afrique de l'Ouest (PPAAO/WAAPP) pour avoir soutenu financièrement cette étude.

\section{RESUME}

Avec l'essor de nouveaux bassins de production maraîchère et la mise en place de fermes agricoles, le maraîchage en Basse Casamance est devenu une activité génératrice de revenus. Les insectes ravageurs et les acariens limitent la production des spéculations par leurs dégâts. Les études effectuées sur l'entomofaune des cultures maraîchères en Basse Casamance sont peu nombreuses et les plus récentes datent de plus de deux décennies. Ce travail de réactualisation est réalisé afin d'avoir une situation de référence qui aidera à proposer des stratégies de gestion des ravageurs. L'inventaire effectué sur une année a permis de prospecter quatre familles de cultures maraîchères que sont les Solanaceae, les Cucurbitaceae, les Brassicaceae et les Malvaceae. Cette étude a permis de recenser 38 genres avec 35 espèces de ravageurs répartis en 17 familles d'insectes et une famille d'acariens. Elle a permis également d'obtenir 13 genres avec 11 espèces d'auxiliaires réparties en 09 familles différentes. Ce résultat est une première étape de mise en place d'une base de données qui peut servir de guide à la prise de décisions pour un meilleur contrôle des populations de nuisibles.

(C) 2017 International Formulae Group. All rights reserved.

Mots clés : Biodiversité, ravageurs, auxiliaires, spéculation, Ziguinchor, Sénégal.

\section{Entomofauna of Vegetable Crops in Lower Casamance (Senegal): Updating}

\begin{abstract}
With the development of new vegetable production areas and the establishment of modern farms, vegetable cropping has become an income generating activity in Lower Casamance. However, insect pests and mites hinder the production due to their great damage to the crops. Research studies on the entomofauna of vegetable crops in Lower Casamance are very few and the most recent date back over two decades. The purpose of this updating is to provide a baseline condition for better pest management strategies. The survey, conducted over a year, led to the prospection of four vegetable families which are Solanaceae, Cucurbitaceae,
\end{abstract}


Brassicaceae and Malvaceae. As a result of this study, 38 genera including 35 species of insect pests belonging to 17 families were recorded. The study also made it possible to identify 13 genera and 11 species of beneficial insects belonging to 9 different families. This result is a first step in setting up a database that can serve as a baseline support decision-making for a better control of pest populations.

(C) 2017 International Formulae Group. All rights reserved.

Key words: Biodiversity, pests, beneficial insects, speculation, Ziguinchor, Senegal.

\section{INTRODUCTION}

Au Sénégal, le maraîchage occupe une place très importante et se déroule suivant deux périodes, la contre saison froide d'octobre à mars et la contre saison chaude d'avril à juin (ANSD, 2014). Selon l'Agence Nationale de la Statistique et de la Démographie, la contre saison fraîche mobilise 53004 ménages et la région de Ziguinchor en compte le plus grand nombre dans le secteur, juste derrière Thiès et $\mathrm{St}$ Louis. Les spéculations les plus cultivées sont l'oignon (40,1\%), le piment $(35,5 \%)$, l'aubergine africaine $(28,4 \%)$, le chou $(27,2 \%)$ et l'aubergine douce $(26,3 \%)$. En contre saison chaude, 29837 ménages sont mobilisés de nouveau derrière Thiès et St. Louis. Les spéculations les plus cultivées restent toujours l'oignon $(38,8 \%)$, le piment $(37,3 \%)$, la tomate $(36,5 \%)$, l'aubergine africaine $(28,9 \%)$, le gombo $(27,5 \%)$, l'aubergine douce (27\%) et le chou (26,3\%). En Basse Casamance, avec l'essor de nouveaux bassins de production maraîchère et la mise en place de fermes agricoles, le maraîchage est devenu une des principalesactivités génératrices de revenus. Les arthropodes ravageurs posent souvent de véritables problèmes aux producteurs par les dégâts occasionnés (Diatte et al., 2016 ; Labou et al., 2016). L'emploi des pesticides est la méthode de lutte la plus utilisée contre ces ravageurs, avec souvent des conséquences néfastes sur l'environnement et le développement de souches résistantes chez les ravageurs (Gomgnimbou et al., 2009). De ce fait, l'effet bénéfique qui est attendu de l'usage des insecticides peut être contre balancé par la résistance des ravageurs vis-à- vis de ces produits chimiques, du fait de la réduction des populations d'ennemies naturels (Bommarco et al., 2011). Or, ces derniers constituent une faune auxiliaire qui joue un rôle important dans la régulation des ravageurs (Breitenmoser et Baur, 2013). Les ravageurs des cultures maraîchères et leurs ennemies naturels ont fait l'objet de peu d'études en Basse Casamance. Les réactualisations sont très rares voire inexistantes. Les seuls travaux effectués sont ceux de Collingwood et al. (1980) et Etienne et al. (1993). La grande majorité des études récentes a été effectuée sur les ravageurs des cultures fruitières comme la mangue (Vayssières et al., 2012 ; Ndiaye et al., 2015 ; Konta et al., 2015). Les études de Etienne et al. (1993) ont montré que pour les cultures maraîchères, les relevés réalisés sur les ravageurs étaient incomplets et qu'il fallait obtenir des données supplémentaires. Malheureusement depuis lors, presqu'aucune étude sur les ravageurs des cultures maraîchères et leurs auxiliaires n'a été effectuée. Selon Choudourou et al. (2012), l'étape préliminaire pour réussir la protection des cultures est la connaissance des ravageurs. A cela, il faut associer la connaissance de la faune auxiliaire qui participe à la régulation des nuisibles. L'objectif de cette étude est de contribuer à la connaissance de la faune entomologique des cultures maraîchères par la collecte et l'identification des différents ravageurs présents et de leurs auxiliaires.

\section{MATERIEL ET METHODES}

Présentation du milieu d'étude

L'étude a été effectuée dans la région de Ziguinchor (Basse Casamance). Elle est 
située à $12^{\circ} 33^{\prime}$ latitude Nord et $16^{\circ} 16^{\prime}$ de longitude Ouest, déclinaison magnétique $13^{\circ} 05$. Son altitude est de $19,30 \mathrm{~m}$ dans la partie Sud-Ouest du Sénégal. Elle occupe une superficie de $7339 \mathrm{~km}^{2}$ soit $3,73 \%$ du territoire national et est limitée au Nord par la République de Gambie, au Sud par la République de Guinée Bissau, à l'Est par les régions de Kolda et Sédhiou et à l'Ouest par l'Océan Atlantique (ANSD, 2015). La région est influencée par le climat sub-guinéen, favorisant ainsi une forte pluviométrie.La pluviométrie moyenne annuelle peut aller jusqu'à $1500 \mathrm{~mm}$.

\section{Méthodes}

Échantillonnage, élevage et conservation des insectes collectés

L'inventaire des arthropodes ravageurs a été effectué à partir des principales cultures maraîchères. Leur prélèvement se faisait sur tout le plant. Seuls les insectes ayant été trouvés sur les organes (tiges, feuilles, fruits, fleurs) des plantes sont capturés. Les chenilles collectées sont mises individuellement dans des boîtes en plastique. Les fruits piqués des Cucurbitaceae et des Solanaceae sont déposés dans des pots contenant du sable et recouverts de tissus moustiquaires. Les pupes obtenues à partir des fruits attaqués sont suivies jusqu'à l'émergence des adultes. Les individus récoltés sont ramenés au laboratoire et nourris avec des feuilles fraîches et/ou des fruits provenant de leur plante hôte. Le travail s'est déroulé de mars 2015 à décembre 2016. Pour chaque stade phénologique des cultures (végétatif, floraison, fructification et maturation), des visites régulières sont effectuées dans les périmètres maraîchers et/ou dans les zones de fortes productions maraîchères. Les chenilles collectées ont été élevées au Laboratoire d'Entomologie de l'Institut Sénégalais de Recherches Agricoles de Djibélor, jusqu'à l'émergence des adultes ou des parasitoïdes. Les adultes des ravageurs collectés sont immédiatement introduits dans un flacon contenant un papier absorbant et de l'acétate d'éthyleafin de les tuer et faciliter la conservation et leur identification. Après 48 heures, les arthropodes sont étalés sur des couches en coton. La camera "DinoCapture » version 2.0, a été utilisée pour mieux visualiser et photographier les insectes dans leur milieu naturel et dans le laboratoire.

\section{Identification des spécimens}

Le catalogue "des Arthropodes des cultures légumières d'Afrique de l'Ouest, Centrale, Mayotte et Réunion » (Bordat et Arvanitakis, 2004) a été utilisé dans un premier temps. Dans un second temps, la clé de reconnaissance des familles de Delvare et Aberlenc (1989) a été utilisée pour l'identification des espèces. Certains auxiliaires ont été envoyés en France pour identification. Les Tachinidae sont encours d'identification alors que les Braconidae ont été identifiés par Pascal Rousse Taxonomiste international.

\section{RESULTATS}

L'inventaire préliminaire effectué sur les spéculations a permis de faire un recensement des ravageurs et des auxiliaires des cultures maraîchères en Basse Casamance. Au total, 17 familles d'insectes et une famille d'acariens, dont 38 genres et 35 espèces ont été inventoriés pour les ravageurs (Tableau 1). Pour les auxiliaires, 09 familles dont 13 genres avec 11 espèces ont été obtenus (Tableau 2). L'ordre des Hemiptera est le plus représentatif avec 6 familles et 13 genres dont 10 espèces, suivi de l'ordre des Lepidoptera avec 5 familles et 12 espèces, l'ordre des Coleoptera avec 3 familles et 8 genres et de l'ordre des Diptera avec 2 familles et 3 genres. Les ordres les moins représentatifs sont les Acari et les Thysanoptera avec chacun, une famille et un genre. 
Tableau 1 : Identification des arthropodes ravageurs inventoriés sur les principales cultures maraîchères en Basse Casamance.

\begin{tabular}{|c|c|c|c|}
\hline Ordres & Familles & Genres et espèces & Plantes hôtes \\
\hline Acari & Tetranichidae & Tetranychus evansi Baker et Pritchard & Tomate \\
\hline \multirow[t]{8}{*}{ Coleoptera } & Coccinellidae & Henosepilachna reticulata (Olivier) & Courgette, pastèque \\
\hline & Chrysomelidae & Aulacophora foveicollis Lucas & Courgette \\
\hline & & Podagrixena decolorata (Duvivier) & Gombo \\
\hline & & Nisotra dilecta (Dalman) & Gombo \\
\hline & & Phyllotreta cheiranthi Weise & Chou \\
\hline & & Leptaulaca fissicolis Thomson & Courgette \\
\hline & & Diacantha kraatzi (Jacoby) & Courgette \\
\hline & Meloidae & Hycleus senegalensis (Voigts) & Gombo \\
\hline \multirow[t]{3}{*}{ Diptera } & Agromyzidae & Liriomysa sp. & Tomate \\
\hline & Tephritidae & Ceratitis capitata (Wiedemann) & Piment, poivron \\
\hline & & Bactrocera cuccurbitae (Coquillett) & Concombre, pastèque \\
\hline \multirow[t]{13}{*}{ Hemiptera } & Aleyrodidae & Bemisia tabaci (Gennadius) & tomate \\
\hline & Aphididae & Aphis gossypii Glover & Gombo \\
\hline & & Lipaphis pseudobrassicae (Davis) & Chou \\
\hline & Cicadellidae & Cicadelles sp1 ? & Courgette \\
\hline & & Jacobiasca hybrida Begevin \& Zanon? & Aubergine, tomate \\
\hline & & Cicadelles sp2 ? & Gombo \\
\hline & Coreidae & Acanthocoris collarti Scouteden & Aubergine \\
\hline & & Anoplocnemis curvipes (F.) & Gombo \\
\hline & & Cletus spp. & Gombo \\
\hline & & Leptoglossus autralis (F.) & Concombre \\
\hline & Pentatomidae & Aspavia armigera $(\mathrm{F})$. & Gombo \\
\hline & & Nezara viridula (L.) & Concombre, courgette \\
\hline & Pseudococcidae & Cochenilles & Gombo, aubergine \\
\hline \multirow[t]{12}{*}{ Lepidoptera } & Arctiidae & Amsacta moloneyi (Druce) & Chou \\
\hline & Gelechiidae & Tuta absoluta (Meyrick) & Tomate \\
\hline & Noctuidae & Chrysodeixis chalcites (Esper) & Tomate, chou \\
\hline & & Earias biplaga (Walker) & Gombo \\
\hline & & Helicoverpa armigera (Hübner) & Tomate, gombo \\
\hline & & Selepa docilis Butler & Aubergine \\
\hline & & Spodoptera littoralis (Boisduval) & Chou \\
\hline & Plutellidae & Plutella xylostella (L.) & Chou \\
\hline & Pyralidae & Crocidolomia binotalis Zeller & Chou \\
\hline & & Diaphania indica (Saunders) & Concombre, courgette \\
\hline & & Hellula undalis (F.) & Chou \\
\hline & & Sillepte derogata $(\mathrm{F})$. & Gombo \\
\hline Thysanoptera & Thripidae & Thrips tabaci (Lindemann) & Gombo \\
\hline
\end{tabular}


Tableau 2 : Identification des différents auxiliaires obtenus.

\begin{tabular}{|c|c|c|c|c|}
\hline Ordres & Familles & Statut & Genres et Espèces & Ravageurs cibles \\
\hline Mantoda & Mantidae & Prédateur & Mantis religiosa $(\mathrm{L})$. & Arthropodes divers \\
\hline \multirow{3}{*}{ Diptera } & Syrphidae & Prédateur & Ichiodon aegyptius Wiedmann & Pucerons \\
\hline & Tachinidae & Parasitoïde & Sp 1 & A. moloneyi \\
\hline & & Parasitoïde & Sp 2 & H. armigera \\
\hline \multirow[t]{2}{*}{ Hemiptera } & Miridae & Prédateur & Nesidiocoris tenuis (Reuter) & œufs d'arthropodes \\
\hline & Reduviidae & Prédateur & Cosmolestes pictus (Klug) & P. decolorata \\
\hline \multirow[t]{7}{*}{ Hymenoptera } & Braconidae & Parasitoïde & Schoenlandella testacea (Kriechbaumer) & P. indica \\
\hline & Chalcididae & Parasitoïde & Chalcidiens grégaires & C. chalcites \\
\hline & & Parasitoïde & Brachymeria sp. & C. chalcites \\
\hline & Eulophidae & Parasitoïde & Euplectrus sp. & C. chalcites \\
\hline & Ichneumonidae & Parasitoïde & Diadegma insulare (Cresson) & P. xylostella \\
\hline & & Parasitoïde & Charops flavipes (Kriechbaumer). & S. littoralis \\
\hline & & Parasitoïde & Pristomerus pallidus (Brullé). & H. undalis \\
\hline
\end{tabular}

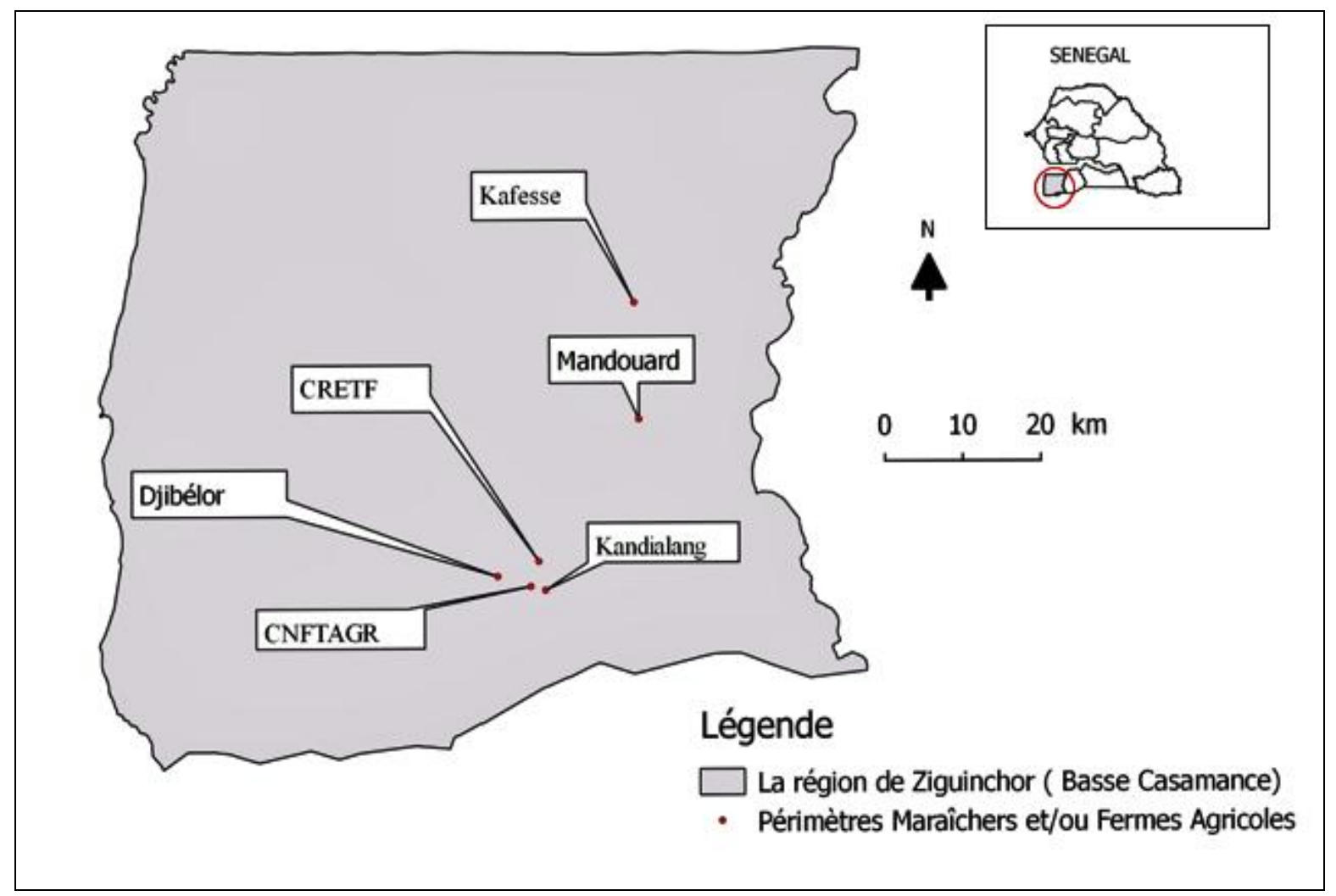

Figure 1 : Carte du milieu d'étude et des sites d'échantillonnage. 


\section{DISCUSSION}

Cette première réactualisation de l'entomofaune des cultures maraîchères de la Basse Casamance a permis d'apporter des compléments sur les relevés effectués précédemment (Collingwood et al., 1980; Etienne et al., 1993). Après identification, 38 genres et 35 espèces d'insectes ont été inventoriés ainsi qu'une famille d'acariens. Une liste non exhaustive des ravageurs et leurs auxiliaires sontproposés (Tableau 1 et Tableau 2). Certains ravageurs n'ont pas été signalés par les travaux d'Etienne et al. (1993). Il s'agit des espèces $C$. chalcites et $A$. moloneyi sur chou, alors que leurs dégâts ont forcé certains producteurs à abandonner leurs parcelles. Signalé comme ravageur des céréales sèches (mil, maïs, niébé), l'espèce $A$. moloneyi s'attaque aussi aux Brassicaceae comme le chou. Les chenilles collectées ont permis d'obtenir des parasitoïdes non identifiés de la famille des Tachinidae. Sur les Solanaceae, les espèces T. evansi, T. absoluta, $B$. tabaci et $C$. chalcites ont été retrouvées sur tomate, alorsqu'ellesn'avaient pas été signalées lors de l'inventaire effectué par Etienne et al. (1993).Les ravageurs des Cucurbitaceae : B. cucurbitae, L. australis et $P$. indica ont aussi causé des problèmes à la production de concombre et de pastèque. La faible production de la pastèque et du concombre en Basse Casamance s'explique par les dégâts essentiellement dus à l'espèce $B$. cucurbitae qui décourage un grand nombre de producteurs. Selon l'ANSD (2014), les ménages impliqués dans la production de pastèque sont peu nombreux (96), comparés à ceux impliqués dans la production du chou (1 416), de gombo (2 198), de l'aubergine africaine $(2$ 116) et de l'aubergine douce (1 584). La faune d'auxiliaires inventoriés est largement dominée par les hyménoptères parasitoïdes surtout ceux de la famille des Braconidae. Ces entomophages jouent un rôle important dans la régulation naturelle des ravageurs. L'espèce Diadegma insulare (Hymenoptera Ichneumonidae) a été signalée dans la zone des Niayes comme parasitoïde de P. xylostella dès 2014 ( Labou et al., 2016). Par contre pour les espèces Charops flavipes (Ichneumonidae Campopleginae) (Kriechbaumer) et Pristomerus pallidus (Ichneumonidae Cremastinae) (Brullé) qui sont encore non signalées dans les Niayes ont été retrouvées respectivement comme parasitoïdes de chenilles de $S$. littoralis et de H. undalis en Basse Casamance. L'espèce Euplectrus sp. (Eulophidae) est retrouvée comme parasitoïde de chenille de $C$. chalcites de même que certains hyménoptères chalcidiens non identifiés. Des diptères Tachinidae ont aussi parasité les chenilles de lépidoptères (A. moloneyi, $H$. armigera), les dégâts de l'espèce $A$. moloneyi ont été signalés au Sénégal par plusieurs études et sur différentes plantes hôtes (haricot, mil, maïs, arachide, coton...) (Cissé et Hall 2001; Ahuja, 2010). Parmi toutes ces études, aucune d'entre elle n'a montré la présence de Tachinidae parasitoïdes des chenilles de A. moloneyi. Par contre, ce travail de réactualisation a permis d'obtenir des Tachnidae parasitoïdes non encore identifiés. Les hémiptères Reduviidae et Miridae (respectivement $C$. pictus et $N$. tenuis), les Dictyoptères Mantidae ( $M$. religiosa) et les diptères Syrphidae ( $I$. aegyptius) sont les seuls prédateurs inventoriés. Les Miridae sont de petits prédateurs hémiptères qui ont en général un régime mixte, mais où prédomine suivant les espèces, soit l'alimentation animale ou l'alimentation végétale (Vayssières et al., 2001). L'espèce $M$. religiosa est un prédateur généraliste qui s'attaque surtout aux insectes adultes, alors que I. aegyptius exerce une action prédatrice sur les larves et les adultes de pucerons. 


\section{Conclusion}

Cette étude a permis de réactualiser pour la première fois depuis vingt ans, la liste des ravageurs des cultures maraîchères et de leurs auxiliaires en Basse Casamance. Elle a montré une très grande diversité de la faune des arthropodes ravageurs et de leurs auxiliaires. Cette étude est une première étape de mise en place d'une base de données qui apporterait des informations utiles avant le choix d'une stratégie de lutte contre les ravageurs. Il est donc important d'élargir la zone d'intervention pour avoir une meilleure connaissance de l'ensemble des cultures maraîchères dans toute la Casamance.

\section{CONFLIT D'INTERETS}

Les auteurs déclarent qu'il n'existe aucun conflit d'intérêts et que l'ordre a été fait à l'unanimité.

\section{CONTRIBUTIONS DES AUTEURS}

ET a été l'investigateur principal ; SD et $\mathrm{KD}$ ont co-encadré les travaux de terrain et ont participé à la rédaction ; BL a participé à la mise en place du protocole et à la rédaction du manuscrit.

\section{REMERCIEMENTS}

Nous remercions le Dr. Dominique Bordat (Entomologiste) pour l'aide apportée à la rédaction de ce document et à l'identification de certaines espèces récoltées. Nous remercions M. Landing Diassy, Technicien du Laboratoire d'Entomologie de Djibélor (ISRA) pour son aide ainsi que les étudiants du Département d'Agroforesterie de l'Université AssaneSeck de Ziguinchor pour l'aide apportée lors des récoltes aux champs.

\section{REFERENCES}

Ahuja SL. 2010. Research and Development on Cotton during and after the IndianSupported Agricultural Development Project in Senegal. Outlook on
Agriculture, 39(3): 211-215. DOI: doi:10.5367/oa.2010.0009.

ANSD (Agence Nationale de la Statistique et de la Démographie).2014. Recensement Général de la Population et de l'Habitat, de l'Agriculture et de l'Elevage (RGPHAE). 2013. ANSD, 372p.

ANSD (Agence Nationale de la Statistique et de la Démographie). 2015. Situation économique et sociale régionale 2013. Service Régional de la Statistique et de la Démographie de Ziguinchor.SRSDZ $102 \mathrm{p}$.

Bommarco R, Miranda F, BylundH, Björkman C. 2011. Insecticides Suppress Natural Enemies and Increase Pest Damage in Cabbage. J. Econ. Entomol., 104(3): 782-791. DOI: 10.1603/EC10444.

Bordat D, Arvanitakis L. 2004. Arthropodes des cultures légumières d'Afrique de l'Ouest, centrale, Mayotte et Réunion. CIRAD-FLHOR., 291p.

Breitenmoser S, Baur R. 2013. Influence des insecticides sur les auxiliaires dans les céréales et pommes de terre. Rech. Agron.Suisse., 4(9): 376-383.

Choudourou DC, Agbaka A, Adjakpa, JB, Koutchika, RE, Adjalian EJN. 2012. Inventaire préliminaire de l'entomofaune des champs de tomates (Lycopersicon esculentum Mill) dans la Commune de Djakotomey au Bénin. Int. J. Biol. Chem. Sci., 6(4): 1798-1804. DOI: http://dx.doi.org/10.4314/ijbcs.v6i4.34

Cissé N, Hall AE. 2001. «La Culture Traditionnelle du Niébé au Sénégal, Etude de Cas ». ISRA/CNRA BP 53: 92521-0124

Collingwood EF, Bourdouxhe L, Dubern J, 1980. Mission d'étude des problèmes phytosanitaires des cultures maraichères en Casamance (24-26 mars 1980): rapport de mission. ORSTOM/ISRA,10p. multigr. 
http://www.documentation.ird.fr/hor/fdi: 33277.

Delvare G, Aberlenc HP. 1989. Les insectes d'Afrique et d'Amérique Tropicale.Clé pour la Reconnaissance des Familles. Labo de Faunistique, Département GERDAT : Montpellier, France.298p.

Diatte M, Brévault T, Sall-Sy D, Diarra K. 2016. Des pratiques culturales influent sur les attaques de deux ravageurs de la tomate dans les Niayes au Sénégal. Int. J. Biol. Chem. Sci., 10(2): 681-693. DOI: http://dx.doi.org/10.4314/ijbcs.v10i2.19

Etienne J, Delvare G, Aberlenc, HP. 1993. Contribution to the knowledge of arthropods associated with cultivated plants in Casamance (Senegal). Boll. Zool. agr. Bachic. Ser.II., 24 (2): 159193.

Gomgnimbou AP, Savadogo PW, Nianogo AJ, Millogo-Rasolodimby J. 2009. Usage des intrants chimiques dans un agrosystème tropical: diagnostic $\mathrm{du}$ risque de pollution environnementale dans la région cotonnière de l'est du Burkina Faso. Biotechnol. Agron. Société Environ., 13(4): 499-507.

Konta IS, Djiba S, Sane S, Diassi L, Ndiaye AB, Noba K. 2015. Etude de la dynamique de Bactrocera dorsalis (Hendel) (Diptera: Tephritidae) dans les vergers de mangues en Basse Casamance: influence des facteurs climatiques. Int. J. Biol. Chem. Sci., 9(6): 2698-2715.

DOI:

http://dx.doi.org/10.4314/ijbcs.v9i6.15
Labou B, Bordat D, Brevault T, Diarra K. 2016. Importance de la" Teigne du chou" dans les Niayes au Sénégal: interrelations avec la température et les cultivars utilisés. Int. J. Biol. Chem. Sci., 10(2): 706-721. DOI : http://dx.doi.org/10.4314/ijbcs.v10i2.21

Labou B, Bordat D, Niang AA, Diarra K. 2016. First record of the larval parasitoid Diadegma insulare (Cresson) (Hymenoptera: Ichneumonidae: Campopleginae) from Senegal. Afr. Entomol., 24(2) 533-535. DOI: 10.4001/003.024.0533

Ndiaye O, Ndiaye S, Djiba S, Ba CT, Vaughan L, ReyJY, Vayssières JF. 2015. Preliminary surveys after release of the fruit fly parasitoid Fopius arisanus Sonan (Hymenoptera Braconidae) in mango production systems in Casamance (Senegal). Fruits., 70(2): 91-99. DOI: $10.1051 /$ fruits/2015001.

Vayssières JF, Adandonon A, N'Diaye O, Sinzogan A, Kooymann C, Badji K, Rey JY, Wharton RA. 2012. Native parasitoids associated with fruit flies (Diptera: Tephritidae) in cultivated and wild fruit crops in Casamance, Senegal. Afr. Entomol., 20(2): 308-315. DOI: 10.4001/003.020.0221.

Vayssières JF, Delvare G, Maldes JM, Aberlenc HP. 2001. Inventaire préliminaire des arthropodes ravageurs et auxiliaires des cultures maraîchères sur l'Ile de la Réunion. Int. J. Trop. Insect Sci., 21: 1-22. 